\title{
Singularities of attractive and repulsive type for $p$-Laplacian generalized Liénard equation
}

Yun Xin ${ }^{1}$ and Hongmin Liu ${ }^{1 *}$

\section{*Correspondence:}

hongminliu_1982@126.com

${ }^{1}$ College of Computer Science and

Technology, Henan Polytechnic

University, Jiaozuo, China

\begin{abstract}
This paper is devoted to an investigation of a kind of $p$-Laplacian generalized Liénard equations with singularities of attractive and repulsive type, where the nonlinear term $g$ has a singularity at the origin. The novelty of the present article is that we show that singularities of attractive and repulsive type enable the achievement of a new existence criterion of a positive periodic solution through an application of the Manásevich-Mawhin theorem on continuity of the topological degree, recent results in the literature are generalized and significantly improved. Finally, some examples are given to show applications of the theorems.
\end{abstract}

MSC: 34B16; 34C25

Keywords: Positive periodic solution; $p$-Laplacian; Singularity of attractive type; Singularities of repulsive type; Generalized Liénard equation

\section{Introduction}

In this paper, we consider the following $p$-Laplacian generalized Liénard equation with singularity:

$$
\left(\phi_{p}\left(x^{\prime}(t)\right)\right)^{\prime}+f(t, x(t)) x^{\prime}(t)+g(t, x(t))=e(t),
$$

where $p \geq 2, \phi_{p}(x)=|x|^{p-2} x$ for $x \neq 0$ and $\phi_{p}(0)=0, f: \mathbb{R} \times \mathbb{R} \rightarrow \mathbb{R}$ is a continuous function and it is $T$-periodic about $t, e \in C(\mathbb{R}, \mathbb{R})$ is a $T$-periodic function, $g(t, x)=g_{1}(t, x)+g_{0}(x)$, $g_{1}: \mathbb{R} \times \mathbb{R} \rightarrow \mathbb{R}$ is a continuous function and it is $T$-periodic about $t, g_{0}:(0,+\infty) \rightarrow \mathbb{R}$ is a continuous function and has a singularity at the origin, i.e.,

$$
\lim _{x \rightarrow 0^{+}} g_{0}(x)=+\infty \quad\left(\text { or } \lim _{x \rightarrow 0^{+}} g_{0}(x)=-\infty\right) .
$$

It is said that (1.1) is singularity of attractive type (resp. repulsive type) if $g_{0}(x) \rightarrow+\infty$ (resp. $g_{0}(x) \rightarrow-\infty$ ) as $x \rightarrow 0^{+}$.

The Liénard equation [10],

$$
x^{\prime \prime}+f(x) x^{\prime}+g(x)=0
$$

(c) The Author(s) 2018. This article is distributed under the terms of the Creative Commons Attribution 4.0 International License (http://creativecommons.org/licenses/by/4.0/), which permits unrestricted use, distribution, and reproduction in any medium, provided you give appropriate credit to the original author(s) and the source, provide a link to the Creative Commons license, and indicate if changes were made. 
appears as a simplified model in many domains in science and engineering. It was intensively studied during the first half of the 20th century as it can be used to model oscillating circuits or simple pendulums. For example, the Van der Pol oscillator

$$
x^{\prime \prime}-\mu\left(1-x^{2}\right) x^{\prime}+x=0
$$

is a Liénard equation.

From then on, there has appeared some good amount of work on periodic solutions for Liénard equations and the references cited therein. Some classical tools have been used to study the Liénard equation in the literature, including topological degree methods $[12,17]$, Mawhin's coincidence degree theorem [1, 3, 4, 11], Massera's theorem [21], the Manásevich-Mawhin theorem on continuity of the topological degree [23, 26], Schauder's fixed point theorem [20], generalized polar coordinates [22], and the Poincaré map [27].

At the same time, the study of periodic solution of the Liénard equation with singularity can be traced back to 1996. Zhang in [29] discussed the following singular Liénard equation:

$$
x^{\prime \prime}+f(x) x^{\prime}+g(t, x)=0
$$

where the nonlinear term $g$ has a singularity of repulsive type. The author showed that Eq. (1.3) has at least one periodic solution by applications of coincidence degree theory. Zhang's work has attracted the attention of many scholars in differential equations and they have contributed to the research of Liénard equation with singularity of repulsive type (see, e.g., [2, 5, 6, 8, 9, 13-16, 19, 24, 25, 28, 30]). For example, Jebelean and Mawhin [6] in 2004 investigated the following quasi-linear equation of $p$-Laplacian type:

$$
\left(\phi_{p}\left(x^{\prime}(t)\right)\right)^{\prime}+f(x) x^{\prime}(t)+g(x)=e(t)
$$

where the nonlinear term $g$ satisfied a slightly stronger singularity, i.e.,

$$
\int_{0}^{1} g(u) d u=-\infty
$$

The authors proven that the above problem has at least one positive periodic solution through a basic application of the Manásevich-Mawhin theorem on continuity of the topological degree. Afterwards, using the Manásevich-Mawhin theorem on continuity of the topological degree again, Lu et al. [15] in 2017 obtained the existence of a positive periodic solution of the following equation with singularity of repulsive type:

$$
x^{\prime \prime}+f(x) x^{\prime}-\frac{\alpha(t)}{x^{\mu}}=e(t) .
$$

All the aforementioned results concern Liénard equations and Liénard equations with singularity of repulsive type. Naturally, a new question arises: how does a generalized Liénard equation work on singularities of attractive and repulsive type? Besides the practical interests, the topic has obvious intrinsic theoretical significance. To answer this question, in this paper, we try to fill this gap and establish the existence of positive periodic solutions 
of Eq. (1.1) with singularities of attractive and repulsive type. Applying the ManásevichMawhin theorem on continuity of the topological degree, we obtain the following conclusions.

Theorem 1.1 Suppose the following conditions hold:

$\left(H_{1}\right)$ There exist constants $0<d_{1}<d_{2}$ such that $g(t, x)-e(t)<0$ for $(t, x) \in[0, T] \times\left(0, d_{1}\right)$ and $g(t, x)-e(t)>0$ for $(t, x) \in[0, T] \times\left(d_{2},+\infty\right)$.

$\left(H_{2}\right)$ There exist positive constants $m$ and $n$ such that

$$
|f(t, x)| \leq m|x|^{p-2}+n, \quad \text { for all }(t, x) \in[0, T] \times \mathbb{R} .
$$

$\left(H_{3}\right)$ There exist positive constants $a$ and $b$ such that

$$
g(t, x) \leq a x^{p-1}+b, \quad \text { for all }(t, x) \in[0, T] \times(0,+\infty) .
$$

$\left(H_{4}\right)$ (Repulsive condition) $\lim _{x \rightarrow 0^{+}} \int_{x}^{1} g_{0}(s) d s=-\infty$.

$\left(H_{5}\right)$ There exists a constant $\alpha>0$ such that $\inf _{x \in \mathbb{R}}|f(t, x)| \geq \alpha>0$.

Then Eq. (1.1) has a positive T-periodic solution if the one of the following conditions is satisfied:

(1) $p=2$ and $\frac{a T^{2}}{2}+(m+n) T<1$;

(2) $p>2$ and $\frac{1}{2^{p-2}}\left(\frac{a T}{2}+m\right) T^{\frac{p}{q}}<1$, here $q=\frac{p}{p-1}$.

Remark 1.2 It is worth mentioning that the friction term $f(x) x^{\prime}(t)$ in Eqs. (1.3), (1.4) and (1.5) satisfy $\int_{0}^{T} f(x(t)) x^{\prime}(t) d t=0$, which is crucial to estimate $a$ priori bounds of a positive periodic solution for these equations. However, in this paper, the friction term $f(t, x) x^{\prime}$ may not satisfy $\int_{0}^{T} f(t, x(t)) x^{\prime}(t) d t=0$. For example, let

$$
f(t, x)=\frac{1}{\pi}\left(\cos ^{2} 4 t+3\right) x^{2}(t)+1
$$

Obviously,

$$
\int_{0}^{\frac{\pi}{4}}\left(\frac{1}{\pi}\left(\cos ^{2} 4 t+3\right) x^{2}(t)+1\right) x^{\prime}(t) d t \neq 0
$$

This implies that our methods to estimate a priori bounds of positive periodic solution for Eq. (1.1) are more complex than Eqs. (1.3), (1.4) and (1.5).

Remark 1.3 From [6, 15, 29], the condition imposed on the external force $e(t)$ is $\int_{0}^{T} e(t) d t=$ 0 . But this is unnecessary. For example, let the external force $e(t)=e^{\cos ^{2} 4 t}$. Obviously, $\int_{0}^{T} e^{\cos ^{2} 4 t} d t \neq 0$. Therefore, our result is more general.

Remark 1.4 If Eq. (1.1) satisfies singularity of attractive type, i.e., $\lim _{x \rightarrow 0^{+}} \int_{x}^{1} g_{0}(s) d s=+\infty$. Obviously, attractive condition and $\left(H_{1}\right),\left(H_{3}\right)$ are in contradiction. Therefore, the above method is no long applicable to the proof of the existence of a positive periodic solution for Eq. (1.1) with singularity of attractive type. Next, we give other conditions to prove the existence of a positive periodic solution for Eq. (1.1) with singularity of attractive type. 
Theorem 1.5 Assume that conditions $\left(H_{2}\right)$ and $\left(H_{5}\right)$ hold. Furthermore, suppose the following conditions hold:

$\left(H_{6}\right)$ There exist constants $0<d_{3}<d_{4}$ such that $g(t, x)-e(t)>0$ for $(t, x) \in[0, T] \times\left(0, d_{3}\right)$ and $g(t, x)-e(t)<0$ for $(t, x) \in[0, T] \times\left(d_{4},+\infty\right)$.

$\left(H_{7}\right)$ (Attractive condition) $\lim _{x \rightarrow 0^{+}} \int_{x}^{1} g_{0}(s) d s=+\infty$.

$\left(H_{8}\right)$ There exist positive constants $\beta$ and $\gamma$ such that

$$
-g(t, x) \leq \beta x^{p-1}+\gamma, \quad \text { for all }(t, x) \in[0, T] \times(0,+\infty) .
$$

Then Eq. (1.1) has a positive T-periodic solution if one of the following conditions is satisfied:

(1) $p=2$ and $\frac{\beta T^{2}}{2}+(m+n) T<1$;

(2) $p>2$ and $\frac{1}{2^{p-2}}\left(\frac{\beta T}{2}+m\right) T^{\frac{p}{q}}<1$.

Besides, if the friction term $f(t, x(t))=f(x(t))$, then Eq. (1.1) is rewritten as

$$
\left(\phi_{p}(x)^{\prime}\right)^{\prime}+f(x(t)) x^{\prime}(t)+g(t, x(t))=e(t) .
$$

Note, if $p=2$ and the external force $e(t) \equiv 0$, the quasi-linear operator $x \mapsto\left(\phi_{p}\left(x^{\prime}\right)\right)^{\prime}$ reduces to the linear operator $x \mapsto x^{\prime \prime}$, then (1.7) is of the differential equation form (1.3). Applying the Manásevich-Mawhin theorem on continuity of the topological degree, we obtain the following conclusions.

Theorem 1.6 Assume that conditions $\left(H_{1}\right),\left(H_{3}\right)$ and $\left(H_{4}\right)$ hold. Then Eq. (1.7) has positive $T$-periodic solution if $\frac{a}{2^{p-1}} T^{1+\frac{p}{q}}<1$.

Remark 1.7 If the external force $\int_{0}^{T} e(t) d t=0$, the result in [24, 29] is included in Theorem 1.6.

Theorem 1.8 Assume that conditions $\left(H_{6}\right),\left(H_{7}\right)$ and $\left(H_{8}\right)$ hold. Then Eq. (1.7) has a positive T-periodic solution if $\frac{a}{2^{p-1}} T^{1+\frac{p}{q}}<1$.

If the nonlinear term $g(t, x(t))=g(x(t))$, then Eq. (1.1) is rewritten as

$$
\left(\phi_{p}(x)^{\prime}\right)^{\prime}+f(t, x(t)) x^{\prime}(t)+g(x(t))=e(t) .
$$

Applying the Manásevich-Mawhin theorem on continuity of the topological degree, we obtain the following conclusions.

Theorem 1.9 Assume that condition $\left(H_{5}\right)$ holds. Furthermore, suppose the following conditions hold:

$\left(H_{1}^{*}\right)$ There exist constants $0<d_{1}^{*}<d_{2}^{*}$ such that $g(x)-e(t)<0$ for $x \in\left(0, d_{1}^{*}\right)$ and $g(x)-$ $e(t)>0$ for $x \in\left(d_{2}^{*},+\infty\right)$.

$\left(H_{4}^{*}\right.$ ) (Repulsive condition) $\lim _{x \rightarrow 0^{+}} \int_{x}^{1} g(s) d s=-\infty$.

Then Eq. (1.8) has a positive T-periodic solution.

Remark 1.10 If the friction term $f(t, x) \equiv f(x)$ and the external force $\int_{0}^{T} e(t) d t=0$, Theorem 4.1 in [25] is included in Theorem 1.9. 
Theorem 1.11 Assume that condition $\left(H_{5}\right)$ holds. Suppose the following conditions hold:

$\left(H_{6}^{*}\right)$ There exist constants $0<d_{3}^{*}<d_{4}^{*}$ such that $g(x)-e(t)>0$ for $x \in\left(0, d_{3}^{*}\right)$ and $g(x)-$ $e(t)<0$ for $x \in\left(d_{4}^{*},+\infty\right)$.

$\left(H_{7}^{*}\right)$ (Attractive condition) $\lim _{x \rightarrow 0^{+}} \int_{x}^{1} g(s) d s=+\infty$.

Then Eq. (1.8) has a positive T-periodic solution.

Remark 1.12 We would like to emphasize that the nonlinear term $g$ satisfies the conditions and the work on estimating a priori bounds of positive periodic solutions for Eq. (1.8) is different [25]. In Theorem 3.1 in [25], the nonlinear term $g$ satisfies condition $\left(H_{3}\right)$, i.e., the semi-linearity condition. In this paper, for the nonlinear term $g$ it has not been required that condition $\left(\mathrm{H}_{3}\right)$ holds, i.e., $g$ may be under a sub-linearity condition, a semi-linearity condition or a super-linearity condition. So, we extend and improve the results in [25].

\section{Periodic solution of Eq. (1.1) with singularities of attractive and repulsive type}

Firstly, we embed (1.1) into the following equation family with a parameter $\lambda \in(0,1]$ :

$$
\left(\phi_{p}\left(x^{\prime}(t)\right)\right)^{\prime}+\lambda f(t, x(t)) x^{\prime}(t)+\lambda g(t, x(t))=\lambda e(t) .
$$

By applications of Theorem 3.1 in [18], we obtain the following result.

Lemma 2.1 Assume that there exist positive constants $E_{1}, E_{2}, E_{3}$ and $E_{1}<E_{2}$ such that the following conditions hold:

(1) We have for each possible periodic solution $x$ to Eq. (2.1) that $E_{1}<x(t)<E_{2}$, for all $t \in[0, T]$ and $\left\|x^{\prime}\right\|<E_{3}$, here $\left\|x^{\prime}\right\|:=\max _{t \in[0, T]}\left|x^{\prime}(t)\right|$.

(2) Each possible solution $C$ to the equation

$$
g(t, C)-\frac{1}{T} \int_{0}^{T} e(t) d t=0
$$

satisfies $E_{1}<C<E_{2}$.

(3) We have

$$
\left(g\left(t, E_{1}\right)-\frac{1}{T} \int_{0}^{T} e(t) d t\right)\left(g\left(t, E_{2}\right)-\frac{1}{T} \int_{0}^{T} e(t) d t\right)<0
$$

Then Eq. (1.1) has at least one T-periodic solution.

\subsection{Proof of Theorem 1.1}

Proof of Theorem 1.1 Firstly, we claim that there exists a point $\xi \in[0, T]$ such that

$$
d_{1} \leq x(\xi) \leq d_{2}
$$

In view of $\int_{0}^{T} x^{\prime}(t) d t=0$, we know that there exist two points $t_{1}, t_{2} \in[0, T]$ such that

$$
x^{\prime}\left(t_{1}\right) \geq 0 \quad \text { and } \quad x^{\prime}\left(t_{2}\right) \leq 0 .
$$


Hence, we have

$$
\phi_{p}\left(x^{\prime}\left(t_{1}\right)\right) \geq 0 \quad \text { and } \quad \phi_{p}\left(x^{\prime}\left(t_{2}\right)\right) \leq 0
$$

Let $t_{3}, t_{4} \in[0, T]$ be, respectively, a global maximum and minimum point of $\phi_{p}\left(x^{\prime}(t)\right)$; clearly, we deduce

$$
\begin{aligned}
& \phi_{p}\left(x^{\prime}\left(t_{3}\right)\right) \geq 0, \quad\left(\phi_{p}\left(x^{\prime}\left(t_{3}\right)\right)\right)^{\prime}=0 . \\
& \phi_{p}\left(x^{\prime}\left(t_{4}\right)\right) \leq 0, \quad\left(\phi_{p}\left(x^{\prime}\left(t_{4}\right)\right)\right)^{\prime}=0 .
\end{aligned}
$$

From condition $\left(H_{5}\right)$, we can see that the friction term $f$ will not change sign for $(t, x) \in$ $[0, T] \times(0,+\infty)$. Without loss of generality, suppose $f(t, x)>0$ for $(t, x) \in[0, T] \times(0,+\infty)$ and upon substitution of Eq. (2.3) into Eq. (2.1), we obtain

$$
-\lambda g\left(t_{3}, x\left(t_{3}\right)\right)+\lambda e\left(t_{3}\right)=\lambda f\left(t_{3}, x\left(t_{3}\right)\right) x^{\prime}\left(t_{3}\right)
$$

Since $\phi_{p}\left(x^{\prime}\left(t_{3}\right)\right)=\left|x^{\prime}\left(t_{3}\right)\right|^{p-2} x^{\prime}\left(t_{3}\right) \geq 0$, we know $x^{\prime}\left(t_{3}\right) \geq 0$. So we get

$$
g\left(t_{3}, x\left(t_{3}\right)\right)-e\left(t_{3}\right) \leq 0
$$

From condition $\left(H_{1}\right)$, we know that

$$
x\left(t_{3}\right) \leq d_{2} .
$$

Similarly, from Eq. (2.4), we see that

$$
g\left(t_{4}, x\left(t_{4}\right)\right)-e\left(t_{4}\right) \geq 0,
$$

and again by condition $\left(H_{1}\right)$,

$$
x\left(t_{4}\right) \geq d_{1} .
$$

$x(t)$ is a continuous function in $(0,+\infty)$, from Eqs. (2.5) and (2.6), we get Eq. (2.2). Then we have

$$
\begin{aligned}
\|x\| & =\max _{t \in[0, T]}|x(t)|=\max _{t \in[\xi, \xi+T]}|x(t)| \\
& =\max _{t \in[\xi, \xi+T]}\left|\frac{1}{2}(x(t)+x(t-T))\right| \\
& =\max _{t \in[\xi, \xi+T]}\left|\frac{1}{2}\left(\left(x(\xi)+\int_{\xi}^{t} x^{\prime}(s) d s\right)+\left(x(\xi)-\int_{t-T}^{\xi} x^{\prime}(s) d s\right)\right)\right| \\
& \leq \max _{t \in[\xi, \xi+T]}\left\{d_{2}+\frac{1}{2}\left(\int_{\xi}^{t}\left|x^{\prime}(s)\right| d s+\int_{t-T}^{\xi}\left|x^{\prime}(s)\right| d s\right)\right\} \\
& \leq d_{2}+\frac{1}{2} \int_{0}^{T}\left|x^{\prime}(s)\right| d s .
\end{aligned}
$$


Multiplying both sides of Eq. (2.1) by $x(t)$ and integrating over the interval $[0, T]$, it is clear that

$$
\begin{aligned}
& \int_{0}^{T}\left(\phi_{p}\left(x^{\prime}(t)\right)\right)^{\prime} x(t) d t+\lambda \int_{0}^{T} f(t, x(t)) x^{\prime}(t) x(t) d t+\lambda \int_{0}^{T} g(t, x(t)) x(t) d t \\
& \quad=\lambda \int_{0}^{T} e(t) x(t) d t .
\end{aligned}
$$

Substituting $\int_{0}^{T}\left(\phi_{p}\left(x^{\prime}(t)\right)\right)^{\prime} x(t) d t=-\int_{0}^{T}\left|x^{\prime}(t)\right|^{p} d t$ into Eq. (2.8), we arrive at

$$
\begin{aligned}
& \int_{0}^{T}\left|x^{\prime}(t)\right|^{p} d t \\
& \quad=\lambda \int_{0}^{T} f(t, x(t)) x^{\prime}(t) x(t) d t+\lambda \int_{0}^{T} g(t, x(t)) x(t) d t+\lambda \int_{0}^{T} e(t) x(t) d t \\
& \quad \leq \int_{0}^{T}|f(t, x(t))|\left|x^{\prime}(t)\right||x(t)| d t+\int_{0}^{T}|g(t, x(t))||x(t)| d t+\int_{0}^{T}|e(t)||x(t)| d t \\
& \quad \leq\|x\| \int_{0}^{T}|f(t, x(t))|\left|x^{\prime}(t)\right| d t+\|x\| \int_{0}^{T}|g(t, x(t))| d t+\|x\| \int_{0}^{T}|e(t)| d t .
\end{aligned}
$$

From condition $\left(H_{2}\right)$, Eq. (2.9) and the Hölder inequality, we can observe that

$$
\begin{aligned}
& \int_{0}^{T}\left|x^{\prime}(t)\right|^{p} d t \\
& \quad \leq m\|x\| \int_{0}^{T}|x(t)|^{p-2}\left|x^{\prime}(t)\right| d t+n\|x\| \int_{0}^{T}\left|x^{\prime}(t)\right| d t+\|x\| \int_{0}^{T}|g(t, x(t))| d t \\
& \quad+\|x\| T^{\frac{1}{2}}\left(\int_{0}^{T}|e(t)|^{2} d t\right)^{\frac{1}{2}} \\
& \quad \leq m\|x\|^{p-1} \int_{0}^{T}\left|x^{\prime}(t)\right| d t+n\|x\| \int_{0}^{T}\left|x^{\prime}(t)\right| d t \\
& \quad+\|x\| \int_{0}^{T}|g(t, x(t))| d t+T^{\frac{1}{2}}\|x\|\|e\|_{2},
\end{aligned}
$$

where $\|e\|:=\left(\int_{0}^{T}|e(t)|^{2} d t\right)^{\frac{1}{2}}$.

Integrating over the interval $[0, T]$ for Eq. (2.1), we conclude that

$$
\int_{0}^{T} f(t, x(t)) x^{\prime}(t) d t+\int_{0}^{T} g(t, x(t)) d t=\int_{0}^{T} e(t) d t
$$

From Eq. (2.11), conditions $\left(H_{2}\right)$ and $\left(H_{3}\right)$, we have

$$
\begin{aligned}
\int_{0}^{T}|g(t, x(t))| d t & =\int_{g(t, x(t)) \geq 0} g(t, x(t)) d t-\int_{g(t, x(t)) \leq 0} g(t, x(t)) d t \\
& =2 \int_{g(t, x(t)) \geq 0} g^{+}(t, x(t)) d t+\int_{0}^{T} f(t, x(t)) x^{\prime}(t) d t-\int_{0}^{T} e(t) d t \\
& \leq 2 a \int_{0}^{T} x^{p-1}(t) d t+2 b T+\int_{0}^{T}|f(t, x(t))|\left|x^{\prime}(t)\right| d t+\int_{0}^{T}|e(t)| d t
\end{aligned}
$$




$$
\begin{aligned}
\leq & 2 a\|x\|^{p-1} T+2 b T+m\|x\|^{p-2} \int_{0}^{T}\left|x^{\prime}(t)\right| d t+n \int_{0}^{T}\left|x^{\prime}(t)\right| d t \\
& +T^{\frac{1}{2}}\left(\int_{0}^{T}|e(t)|^{2} d t\right)^{\frac{1}{2}}
\end{aligned}
$$

where $g^{+}(t, x):=\max \{0, g(t, x)\}$. Substituting Eqs. (2.12) and (2.7) into (2.10), we deduce

$$
\begin{aligned}
& \int_{0}^{T}\left|x^{\prime}(t)\right|^{p} d t \\
& \leq 2 m\|x\|^{p-1} \int_{0}^{T}\left|x^{\prime}(t)\right| d t+2 n\|x\| \int_{0}^{T}\left|x^{\prime}(t)\right| d t+2 a\|x\|^{p} T+2 b T\|x\|+2 T^{\frac{1}{2}}\|x\|\|e\|_{2} \\
& \leq 2 m\left(d_{2}+\frac{1}{2} \int_{0}^{T}\left|x^{\prime}(t)\right| d t\right)^{p-1} \int_{0}^{T}\left|x^{\prime}(t)\right| d t \\
&+2 n\left(d_{2}+\frac{1}{2} \int_{0}^{T}\left|x^{\prime}(t)\right| d t\right) \int_{0}^{T}\left|x^{\prime}(t)\right| d t \\
&+2 a T\left(d_{2}+\frac{1}{2} \int_{0}^{T}\left|x^{\prime}(t)\right| d t\right)^{p}+\left(2 b T+2 T^{\frac{1}{2}}\right)\left(d_{2}+\frac{1}{2} \int_{0}^{T}\left|x^{\prime}(t)\right| d t\right) \\
& \leq \frac{1}{2^{p-2}} m\left(1+\frac{2 d_{2}}{\int_{0}^{T}\left|x^{\prime}(t)\right| d t}\right)^{p-1}\left(\int_{0}^{T}\left|x^{\prime}(t)\right| d t\right)^{p} \\
& \quad+ \frac{1}{2^{p-1}} a T\left(1+\frac{2 d_{2}}{\int_{0}^{T}\left|x^{\prime}(t)\right| d t}\right)^{p}\left(\int_{0}^{T}\left|x^{\prime}(t)\right| d t\right)^{p} \\
&+n\left(\int_{0}^{T}\left|x^{\prime}(t)\right| d t\right)^{2}+\left(b T+T^{\frac{1}{2}}\right) \int_{0}^{T}\left|x^{\prime}(t)\right| d t+2 d_{2}\left(n+b T+T^{\frac{1}{2}}\right)
\end{aligned}
$$

Next, we introduce classical elementary inequality (see (3.10) in [7]), there exists a $\delta(p)>$ 0 which is dependent on $p$ only,

$$
(1+x)^{p} \leq 1+(1+p) x, \quad \text { for } x \in[0, \delta(p)]
$$

Then we consider the following two cases:

Case 1. If $\frac{2 d_{2}}{\int_{0}^{T}\left|x_{1}^{\prime}(t)\right| d t}>\delta(p)$, then it is obvious that

$$
\int_{0}^{T}\left|x_{1}^{\prime}(t)\right| d t<\frac{2 d_{2}}{\delta(p)}
$$

From Eq. (2.7), we deduce

$$
\begin{aligned}
\|x\| & \leq d_{2}+\frac{1}{2} \int_{0}^{T}\left|x^{\prime}(t)\right| d t \\
& \leq d_{2}+\frac{2 d_{2}}{\delta(p)}:=M_{1}^{\prime \prime} .
\end{aligned}
$$

Case 2. If $\frac{2 d_{2}}{\int_{0}^{T}\left|x_{1}^{\prime}(t)\right| d t} \leq \delta(p)$, from Eq. (2.14), we obtain

$$
\left(1+\frac{2 d_{2}}{\int_{0}^{T}\left|x^{\prime}(t)\right| d t}\right)^{p-1} \leq 1+\frac{2 d_{2} p}{\int_{0}^{T}\left|x^{\prime}(t)\right| d t}
$$


and

$$
\left(1+\frac{2 d_{2}}{\int_{0}^{T}\left|x^{\prime}(t)\right| d t}\right)^{p} \leq 1+\frac{2 d_{2}(p+1)}{\int_{0}^{T}\left|x^{\prime}(t)\right| d t} .
$$

Substituting Eqs. (2.17) and (2.18) into (2.13), we have

$$
\begin{aligned}
& \int_{0}^{T}\left|x^{\prime}(t)\right|^{p} d t \leq \frac{1}{2^{p-2}} m\left(1+\frac{2 d_{2} p}{\int_{0}^{T}\left|x^{\prime}(t)\right| d t}\right)\left(\int_{0}^{T}\left|x^{\prime}(t)\right| d t\right)^{p} \\
& +\frac{1}{2^{p-1}} a T\left(1+\frac{2 d_{2}(p+1)}{\int_{0}^{T}\left|x^{\prime}(t)\right| d t}\right)\left(\int_{0}^{T}\left|x^{\prime}(t)\right| d t\right)^{p} \\
& +n\left(\int_{0}^{T}\left|x^{\prime}(t)\right| d t\right)^{2}+\left(b T+T^{\frac{1}{2}}\right) \int_{0}^{T}\left|x^{\prime}(t)\right| d t+N_{1} \\
& =\frac{m}{2^{p-2}}\left(\int_{0}^{T}\left|x^{\prime}(t)\right| d t\right)^{p}+\frac{m d_{2} p}{2^{p-3}}\left(\int_{0}^{T}\left|x^{\prime}(t)\right| d t\right)^{p-1} \\
& +\frac{a T}{2^{p-1}}\left(\int_{0}^{T}\left|x^{\prime}(t)\right| d t\right)^{p} \\
& +\frac{\operatorname{aTd}_{2}(p+1)}{2^{p-2}}\left(\int_{0}^{T}\left|x^{\prime}(t)\right| d t\right)^{p-1}+n\left(\int_{0}^{T}\left|x^{\prime}(t)\right| d t\right)^{2} \\
& +\left(b T+T^{\frac{1}{2}}\right) \int_{0}^{T}\left|x^{\prime}(t)\right| d t+N_{1} 1 \\
& =\frac{1}{2^{p-2}}(2 a T+m)\left(\int_{0}^{T}\left|x^{\prime}(t)\right| d t\right)^{p} \\
& +\frac{1}{2^{p-3}}\left(2 a \operatorname{Td}_{2}(p+1)+m d_{2} p\right)\left(\int_{0}^{T}\left|x^{\prime}(t)\right| d t\right)^{p-1} \\
& +n\left(\int_{0}^{T}\left|x^{\prime}(t)\right| d t\right)^{2}+\left(b T+T^{\frac{1}{2}}\right) \int_{0}^{T}\left|x^{\prime}(t)\right| d t+N_{1}
\end{aligned}
$$

where $N_{1}:=2 d_{2}\left(n+b T+T^{\frac{1}{2}}\right)$. Applying the Hölder inequality, it is easy to verify that

$$
\begin{aligned}
\int_{0}^{T}\left|x^{\prime}(t)\right|^{p} d t \leq & \frac{1}{2^{p-2}}(2 a T+m) T^{\frac{p}{q}} \int_{0}^{T}\left|x^{\prime}(t)\right|^{p} d t \\
& +\frac{1}{2^{p-3}}\left(2 a T d_{2}(p+1)+m d_{2} p\right) T^{\frac{p-1}{q}}\left(\int_{0}^{T}\left|x^{\prime}(t)\right|^{p} d t\right)^{\frac{p-1}{p}} \\
& +n T^{\frac{2}{q}}\left(\int_{0}^{T}\left|x^{\prime}(t)\right|^{p} d t\right)^{\frac{2}{p}}+\left(b T+T^{\frac{1}{2}}\right) T^{\frac{1}{q}}\left(\int_{0}^{T}\left|x^{\prime}(t)\right|^{p} d t\right)^{\frac{1}{p}}+N_{1} .
\end{aligned}
$$

Case (I). If $p>2$ and $\frac{1}{2^{p-2}}\left(\frac{a T}{2}+m\right) T^{\frac{p}{q}}<1$, it is easy to see that there exists a positive $M_{1}^{\prime}$ (independent of $\lambda$ ) such that

$$
\int_{0}^{T}\left|x^{\prime}(t)\right|^{p} d t \leq M_{1}^{\prime}
$$


Substituting Eq. (2.20) into (2.7), and using the Hölder inequality, we see that

$$
\|x\| \leq d_{2}+\frac{1}{2} T^{\frac{1}{q}}\left(\int_{0}^{T}\left|x^{\prime}(t)\right|^{p} d t\right)^{\frac{1}{p}} \leq d_{2}+\frac{1}{2} T^{\frac{1}{q}}\left(M_{1}^{\prime}\right)^{\frac{1}{p}}:=M_{1}^{\prime \prime \prime} .
$$

Take $M:=\max \left\{M_{1}^{\prime \prime}, M_{1}^{\prime \prime \prime}\right\}$, we arrive at

$$
\|x\| \leq M_{1}
$$

In view of $x(0)=x(T)$, there exists a point $t_{0} \in[0, T]$ such that $x^{\prime}\left(t_{0}\right)=0$, while $\phi_{p}(0)=0$. Therefore, from Eqs. (2.12), (2.20), (2.21) and condition $\left(H_{2}\right)$, we have

$$
\begin{aligned}
\left|\phi_{p}\left(x^{\prime}(t)\right)\right| & =\left|\int_{t_{0}}^{t}\left(\phi_{p}\left(x^{\prime}(s)\right)\right)^{\prime} d s\right| \\
& \leq \lambda\left(\int_{0}^{T}|f(t, x(t))|\left|x^{\prime}(t)\right| d t+\int_{0}^{T}|g(t, x(t))| d t+\int_{0}^{T}|e(t)| d t\right) \\
& \leq 2 m\|x\|^{p-2} \int_{0}^{T}\left|x^{\prime}(t)\right| d t+2 n \int_{0}^{T}\left|x^{\prime}(t)\right| d t+2 a\|x\|^{p-1} T+2 b T+2 T^{\frac{1}{2}}\|e\|_{2} \\
& \leq 2 m M_{1}^{p-2} T^{\frac{1}{q}}\left(M_{1}^{\prime}\right)^{\frac{1}{p}}+2 n T^{\frac{1}{q}}\left(M_{1}^{\prime}\right)^{\frac{1}{p}}+2 a M_{1}^{p-1} T+2 b T+2 T^{\frac{1}{2}}\|e\|_{2} \\
& :=M_{2}^{\prime} .
\end{aligned}
$$

Besides, we claim that there exists a positive constant $M_{2}>M_{2}^{\prime}+1$ such that, for all $t \in \mathbb{R}$

$$
\left\|x^{\prime}\right\| \leq M_{2}
$$

In fact, if $x^{\prime}$ is not bounded, there exists a positive constant $M_{2}^{\prime \prime}$ such that

$$
\left\|x^{\prime}\right\|>M_{2}^{\prime \prime}
$$

Then we can get

$$
\left\|\phi_{p}\left(x^{\prime}\right)\right\|=\left\|x^{\prime}\right\|^{p-1} \geq\left(M_{2}^{\prime \prime}\right)^{p-1}
$$

which is a contradiction. So Eq. (2.23) holds.

Case (II). If $p=2$ and $\frac{a T^{2}}{2}+(m+n) T<1$, it is easy to see that there exists a positive $M_{1}^{\prime}$ (independent of $\lambda$ ) such that

$$
\int_{0}^{T}\left|x^{\prime}(t)\right|^{2} d t \leq M_{1}^{\prime}
$$

Similarly, we get $\|x\| \leq M_{1}$, $\left\|x^{\prime}\right\| \leq M_{2}$.

On the other hand, it follows from Eq. (2.1) and $g(t, x)=g_{0}(x)+g_{1}(t, x)$ that

$$
\left.\left(\phi_{p}\left(x^{\prime}(t)\right)\right)^{\prime}+\lambda f(t, x(t)) x^{\prime}(t)\right)+\lambda\left(g_{0}(x(t))+g_{1}(t, x(t))\right)=\lambda e(t) .
$$


Let $t \in[0, \xi]$ be as in Eq. (2.7), for any $\xi \leq t \leq T$. From Eqs. (2.7) and (2.23), we conclude that

$$
x(\xi) \geq d_{1} .
$$

Next, we will show that for any $t \in[\xi, T]$, there exists a constant $D_{1} \in\left(0, d_{1}\right)$, such that each positive periodic solution of (1.1) satisfies

$$
x(t)>D_{1} .
$$

In fact, multiplying both sides of Eq. (2.24) by $x^{\prime}(t)$ and integrating on $[\xi, t]$, we get

$$
\begin{aligned}
\lambda \int_{x(\xi)}^{x(t)} g_{0}(u) d u= & \lambda \int_{\xi}^{t} g_{0}(x(s)) x^{\prime}(s) d s \\
= & -\int_{\xi}^{t}\left(\phi\left(x^{\prime}(s)\right)\right)^{\prime} x^{\prime}(s) d s-\lambda \int_{\xi}^{t} f(s, x(s))\left(x^{\prime}(s)\right)^{2} d s \\
& -\lambda \int_{\xi}^{t} g_{1}(s, x(s)) x^{\prime}(s) d s+\lambda \int_{\xi}^{t} e(s) x^{\prime}(s) d s .
\end{aligned}
$$

Furthermore, we get

$$
\begin{aligned}
\lambda\left|\int_{x(\xi)}^{x(t)} g_{0}(u) d u\right| \leq & \left|\int_{\xi}^{t}\left(\phi\left(x^{\prime}(s)\right)\right)^{\prime} x^{\prime}(s) d s\right|+\lambda\left|\int_{\xi}^{t} f(s, x(s))\left(x^{\prime}(s)\right)^{2} d s\right| \\
& +\lambda\left|\int_{\xi}^{t} g_{1}(s, x(s)) x^{\prime}(s) d s\right|+\lambda\left|\int_{\xi}^{t} e(s) x^{\prime}(s) d s\right| .
\end{aligned}
$$

By Eqs. (2.1), (2.22) and (2.23), we arrive at

$$
\begin{aligned}
\left|\int_{\xi}^{t}\left(\phi_{p}\left(x^{\prime}(t)\right)\right)^{\prime} x^{\prime}(s) d s\right| & \leq\left\|x^{\prime}\right\| \int_{0}^{T}\left|\left(\phi\left(x^{\prime}(s)\right)\right)^{\prime}\right| d s \\
& \leq \lambda\left\|x^{\prime}\right\|\left(\int_{0}^{T}\left|f\left(s, x^{\prime}(s)\right)\right| d s+\int_{0}^{T}|g(s, x(s))| d s+\int_{0}^{T}|e(s)| d s\right) \\
& \leq 2 \lambda M_{2} M_{2}^{\prime} .
\end{aligned}
$$

Moreover, from Eq. (2.25), we deduce

$$
\begin{aligned}
& \left|\int_{\xi}^{t} f(s, x(s))\left(x^{\prime}(s)\right)^{2}\right| \leq\left\|x^{\prime}\right\|^{2} T\left(m\|x\|^{p-2}+n\right) \leq M_{2}^{2} T\left(m M_{1}^{p-2}+n\right), \\
& \left|\int_{\xi}^{t} g_{1}(s, x(s)) x^{\prime}(s) d s\right| \leq\|x\| \int_{0}^{T}\left|g_{1}(s, x(s))\right| d s \leq M_{2} \sqrt{T}\left\|g_{M_{1}}\right\|_{2}, \\
& \left|\int_{\xi}^{t} e(s) x^{\prime}(s) d t\right| \leq M_{2} \sqrt{T}\|e\|_{2},
\end{aligned}
$$

where $\left\|g_{M_{1}}\right\|:=\max _{0<x \leq M_{1}}\left|g_{1}(t, x)\right| \in L^{2}(0, T)$. Form these inequalities we can derive from equation (2.25) that

$$
\left|\int_{x(\xi)}^{x(t)} g_{0}(u) d u\right| \leq M_{2}\left(2 M_{2}^{\prime}+M_{2} T\left(m M_{1}^{p-2}+n\right)+\sqrt{T}\left\|g_{M_{1}}\right\|_{2}+\sqrt{T}\|e\|_{2}\right):=M_{3} .
$$


In view of the repulsive condition $\left(H_{4}\right)$ and $x(\xi) \geq d_{1}$, there exists $D_{1} \in\left(0, d_{1}\right)$ such that

$$
\left|\int_{d_{1}}^{D_{1}} g_{0}(u) d u\right|>M_{3}
$$

Thus, there exists a point $\eta \in[\xi, T]$ such that $x(\eta) \leq D_{1}$, then

$$
\left|\int_{x(\xi)}^{x(\eta)} g_{0}(u) d u\right| \geq\left|\int_{d_{1}}^{D_{1}} g_{0}(u) d u\right|>M_{3}
$$

which contradicts Eq. (2.26). Therefore, we can obtain that

$$
x(t) \geq D_{1}, \quad \text { for all } t \in[\xi, T] .
$$

Similarly, we can consider $t \in[0, \xi]$.

Let $E_{1}<\min \left\{D_{1}, M_{3}\right\}, E_{2}>\max \left\{d_{2}, M_{1}\right\}, E_{3}>M_{2}$ are constants, from Eqs. (2.7), (2.21) and (2.23), we see that periodic solution $x$ to Eq. (2.1) satisfies

$$
E_{1}<x(t)<E_{2}, \quad\left\|x^{\prime}\right\|<E_{3} .
$$

Then condition (1) of Lemma 2.1 is satisfied. For a possible solution $C$ in the equation

$$
g(t, C)-\frac{1}{T} \int_{0}^{T} e(t) d t=0
$$

satisfies $E_{1}<C<E_{2}$. Therefore, condition (2) of Lemma 2.1 holds. Finally, we consider that condition (3) of Lemma 2.1 is also satisfied. In fact, from $\left(H_{1}\right)$, we have

$$
g\left(t, E_{1}\right)-\frac{1}{T} \int_{0}^{T} e(t) d t<0
$$

and

$$
g\left(t, E_{2}\right)-\frac{1}{T} \int_{0}^{T} e(t) d t>0
$$

So condition (3) is also satisfied. Applying Lemma 2.1, we see that Eq. (1.1) has at least one positive periodic solution.

\subsection{Proof of Theorem 1.5}

Proof of Theorem 1.5 Let $\underline{t}, \bar{t}$, respectively, the global minimum and maximum points $x(t)$ on $[0, T]$; then $x^{\prime}(t)=0$ and $x^{\prime}(\bar{t})=0$, and we claim that

$$
\left(\phi_{p}\left(x^{\prime}(\bar{t})\right)\right)^{\prime} \leq 0
$$

In fact, if Eq. (2.27) does not hold, then $\left(\phi_{p}\left(x^{\prime}(\bar{t})\right)\right)^{\prime}>0$ and there exists $\varepsilon>0$ such that $\left(\phi_{p}\left(x^{\prime}(t)\right)\right)^{\prime}>0$ for $t \in(\bar{t}-\varepsilon, \bar{t}+\varepsilon)$. Therefore $\phi_{p}\left(x^{\prime}(t)\right)$ is strictly increasing for $t \in(\bar{t}-\varepsilon, \bar{t}+\varepsilon)$. 
Then we know that $x^{\prime}(t)$ is strictly increasing for $t \in(\bar{t}-\varepsilon, \bar{t}+\varepsilon)$. This contradicts the definition of $\bar{t}$. Thus, equation (2.27) is true. From Eqs. (2.1) and (2.27), we get

$$
g(\bar{t}+x(\bar{t}))-e(\bar{t}) \leq 0
$$

Similarly, we deduce

$$
g(\underline{t}+x(\underline{t}))-e(\underline{t}) \geq 0
$$

By condition $\left(H_{6}\right)$, Eqs. (2.28) and (2.29), we see that

$$
x(\underline{t}) \geq d_{3}, \quad \text { and } \quad x(\bar{t}) \leq d_{4} .
$$

In view of $x$ being a continuous function, we see that there exists a point $\xi^{*} \in(0, T)$ such that

$$
d_{3} \leq x\left(\xi^{*}\right) \leq d_{4}
$$

From Eq. (2.7), we have

$$
x(t) \leq d_{4}+\frac{1}{2} \int_{0}^{T}\left|x^{\prime}(t)\right| d t .
$$

We follow the same strategy and notation as in the proof of Theorem 1.1. From Eqs. (2.11), (2.12), condition $\left(H_{2}\right)$ and $\left(H_{8}\right)$, we obtain

$$
\begin{aligned}
& \int_{0}^{T}|g(t, x(t))| d t \\
& \quad=\int_{g(t, x(t)) \geq 0} g(t, x(t)) d t-\int_{g(t, x(t)) \leq 0} g(t, x(t)) d t \\
& \quad=-2 \int_{g(t, x(t)) \leq 0} g^{-}(t, x(t)) d t-\int_{0}^{T} f(t, x(t)) x^{\prime}(t) d t+\int_{0}^{T} e(t) d t \\
& \quad \leq 2 \beta \int_{0}^{T} x^{p-1}(t) d t+2 \gamma T+\int_{0}^{T}|f(t, x(t))|\left|x^{\prime}(t)\right| d t+\int_{0}^{T}|e(t)| d t \\
& \quad \leq 2 \beta T\|x\|^{p-1}+2 \gamma T+\int_{0}^{T}|f(t, x(t))|\left|x^{\prime}(t)\right| d t+\int_{0}^{T}|e(t)| d t,
\end{aligned}
$$

where $g^{-}(t, x):=\min \{g(t, x), 0\}$. The remaining part of the proof is the same as that of Theorem 1.1.

\section{Periodic solution of Eq. (1.7) with singularities of attractive and repulsive type}

\subsection{Proof of Theorem 1.6}

Proof of Theorem 1.6 Consider the homotopic equation

$$
\left(\phi_{p}\left(x^{\prime}(t)\right)\right)^{\prime}+\lambda f(x(t)) x^{\prime}(t)+\lambda g(t, x(t))=e(t) .
$$


We follow the same strategy and notation as in the proof of Theorem 1.1. From Eq. (2.7), we have

$$
\int_{0}^{T}\left(\phi_{p}\left(x^{\prime}(t)\right)\right)^{\prime} x(t) d t+\lambda \int_{0}^{T} g(t, x(t)) x(t) d t=\lambda \int_{0}^{T} e(t) x(t) d t
$$

since $\int_{0}^{T} f(x(t)) x^{\prime}(t) x(t) d t=0$. Substituting $\int_{0}^{T}\left(\phi_{p}\left(x^{\prime}(t)\right)\right)^{\prime} x(t) d t=-\int_{0}^{T}\left|x^{\prime}(t)\right|^{p} d t$ into equation (3.2), we get

$$
\int_{0}^{T}\left|x^{\prime}(t)\right|^{p} d t \leq\|x\| \int_{0}^{T}|g(t, x(t))| d t+\|x\| \int_{0}^{T}|e(t)| d t
$$

Integrating over the interval $[0, T]$ for Eq. (3.1), we obtain

$$
\int_{0}^{T} g(t, x(t)) d t=\int_{0}^{T} e(t) d t
$$

From Eq. (3.4) and condition $\left(H_{3}\right)$, we see that

$$
\begin{aligned}
\int_{0}^{T}|g(t, x(t))| d t & =\int_{g(t, x(t)) \geq 0} g(t, x(t)) d t-\int_{g(t, x(t)) \leq 0} g(t, x(t)) d t \\
& =2 \int_{g(t, x(t)) \geq 0} g^{+}(t, x(t)) d t-\int_{0}^{T} e(t) d t \\
& \leq 2 a \int_{0}^{T} x^{p-1}(t) d t+2 b T+\int_{0}^{T}|e(t)| d t \\
& \leq 2 a\|x\|^{p-1} T+2 b T+T^{\frac{1}{2}}\left(\int_{0}^{T}|e(t)|^{2} d t\right)^{\frac{1}{2}} .
\end{aligned}
$$

Substituting Eqs. (3.5) into (3.3), and from Eq. (2.19), we conclude that

$$
\begin{aligned}
\int_{0}^{T}\left|x^{\prime}(t)\right|^{p} d t \leq & \frac{2 a T^{1+\frac{p}{q}}}{2^{p-2}} \int_{0}^{T}\left|x^{\prime}(t)\right|^{p} d t+\frac{a d_{2}(p+1) T^{1+\frac{p-1}{q}}}{2^{p-2}}\left(\int_{0}^{T}\left|x^{\prime}(t)\right|^{p} d t\right)^{\frac{p-1}{p}} \\
& +\left(b T+T^{\frac{1}{2}}\right) T^{\frac{1}{q}}\left(\int_{0}^{T}\left|x^{\prime}(t)\right|^{p} d t\right)^{\frac{1}{p}}+N_{1} .
\end{aligned}
$$

Since $\frac{2 a T^{1+\frac{p}{q}}}{2^{p-2}}<1$, it is easy to see that there exists a positive $M_{1}^{\prime}$ (independent of $\lambda$ ) such that

$$
\int_{0}^{T}\left|x^{\prime}(t)\right| d t \leq M_{1}^{\prime}
$$

The remaining part of the proof is the same as that of Theorem 1.1.

\subsection{Proof of Theorem 1.8}

Proof of Theorem 1.8 We follow the same strategy and notation as in the proof of Theorem 1.5 and 1.6. We only consider $\int_{0}^{T}|g(t, x(t))| d t$. 
From Eqs. (2.31), (3.4) and condition $\left(H_{8}\right)$, we have

$$
\begin{aligned}
\int_{0}^{T}|g(t, x(t))| d t & =\int_{g(t, x(t)) \geq 0} g(t, x(t)) d t-\int_{g(t, x(t)) \leq 0} g(t, x(t)) d t \\
& =-2 \int_{g(t, x(t)) \leq 0} g^{-}(t, x(t)) d t+\int_{0}^{T} e(t) d t \\
& \leq 2 \beta \int_{0}^{T} x^{p-1}(t) d t+2 \gamma T+\int_{0}^{T}|e(t)| d t \\
& \leq 2 \beta T\|x\|^{p-1}+2 \gamma T+\int_{0}^{T}|e(t)| d t .
\end{aligned}
$$

The remaining part of the proof is the same as that of Theorem 1.1.

\section{Periodic solution of Eq. (1.8) with singularities of attractive and repulsive type}

\subsection{Proof of Theorem 1.9}

Proof of Theorem 1.9 Consider the homotopic equation

$$
\left(\phi_{p}\left(x^{\prime}(t)\right)\right)^{\prime}+\lambda f(t, x(t)) x^{\prime}(t)+\lambda g(x(t))=e(t) .
$$

We follow the same strategy and notation as in the proof of Theorem 1.1. From Eq. (2.7), we deduce

$$
x(t) \leq d_{2}^{*}+\frac{1}{2} \int_{0}^{T}\left|x^{\prime}(t)\right| d t
$$

Multiplying both sides of Eq. (4.1) by $x^{\prime}(t)$ and integration over the interval $[0, T]$, we get

$$
\begin{aligned}
& \int_{0}^{T}\left(\phi_{p}\left(x^{\prime}(t)\right)\right)^{\prime} x^{\prime}(t) d t+\lambda \int_{0}^{T} f(t, x(t))\left(x^{\prime}(t)\right)^{2} d t+\lambda \int_{0}^{T} g(x(t)) x^{\prime}(t) d t \\
& \quad=\lambda \int_{0}^{T} e(t) x^{\prime}(t) d t .
\end{aligned}
$$

Since $\int_{0}^{T}\left(\phi_{p}\left(x^{\prime}(t)\right)\right)^{\prime} x^{\prime}(t) d t=0$ and $\int_{0}^{T} g(x(t)) x^{\prime}(t) d t=0$, we obtain

$$
\int_{0}^{T} f(t, x(t))\left|x^{\prime}(t)\right|^{2} d t=\int_{0}^{T} e(t) x^{\prime}(t) d t
$$

From Eq. (4.3), we have

$$
\left.\left|\int_{0}^{T} f(t, x(t))\right| x^{\prime}(t)\right|^{2} d t|=| \int_{0}^{T} e(t) x^{\prime}(t) d t \mid
$$

From condition $\left(H_{5}\right)$, we see that

$$
\left.\left.\left|\int_{0}^{T} f(t, x(t))\right| x^{\prime}(t)\right|^{2} d t\left|=\int_{0}^{T}\right| f(t, x(t))|| x^{\prime}(t)\right|^{2} d t \geq \alpha \int_{0}^{T}\left|x^{\prime}(t)\right|^{2} d t .
$$


Substituting Eqs. (4.5) into (4.4), and using the Hölder inequality, we arrive at

$$
\begin{aligned}
\alpha \int_{0}^{T}\left|x^{\prime}(t)\right|^{2} d t & \leq \int_{0}^{T}|e(t)||x(t)| d t \\
& \leq\left(\int_{0}^{T}|e(t)|^{2} d t\right)^{\frac{1}{2}}\left(\int_{0}^{T}|x(t)|^{2} d t\right)^{\frac{1}{2}} \\
& =\|e\|_{2}\left(\int_{0}^{T}|x(t)|^{2} d t\right)^{\frac{1}{2}} .
\end{aligned}
$$

It is easy to see that there exists a positive constant $M_{1}^{\prime}$ (independent of $\lambda$ ) such that

$$
\int_{0}^{T}\left|x^{\prime}(t)\right|^{2} d t \leq M_{1}^{\prime}
$$

From Eq. (2.21), it is obvious that

$$
x(t) \leq M_{1}
$$

Integrating both sides of Eq. (4.1) over $[0, T]$, it is clear that

$$
\int_{0}^{T}\left[f(t, x(t)) x^{\prime}(t)+g(x(t))-e(t)\right] d t=0
$$

From Eq. (4.6), we have

$$
\begin{aligned}
\int_{0}^{T}|g(x(t))| d t & =\int_{g(x(t)) \geq 0} g(x(t)) d t-\int_{g(x(t)) \leq 0} g(x(t)) d t \\
& =2 \int_{g(x(t)) \geq 0} g^{+}(x(t)) d t+\int_{0}^{T} f(t, x(t)) x^{\prime}(t) d t-\int_{0}^{T} e(t) d t .
\end{aligned}
$$

Case (I). If $\bar{e}:=\frac{1}{T} \int_{0}^{T} e(t) d t \leq 0$, from (4.7), we get

$$
\int_{0}^{T}|g(x(t))| d t \leq 2 \int_{0}^{T}\left(g^{+}(x(t))-e(t)\right) d t+\int_{0}^{T} f(t, x(t)) x^{\prime}(t) d t .
$$

Since $g^{+}(x(t))-e(t) \geq 0$, from condition $\left(H_{1}^{*}\right)$, we know $x(t) \geq d_{2}^{*}$. Then we deduce

$$
\begin{aligned}
\int_{0}^{T}|g(x(t))| d t & \leq 2 \int_{0}^{T} g^{+}(x(t)) d t+\int_{0}^{T}|f(t, x(t))|\left|x^{\prime}(t)\right|+\int_{0}^{T}|e(t)| d t \\
& \leq 2 T\left\|g_{M_{1}}^{+}\right\|+\int_{0}^{T}|f(t, x(t))|\left|x^{\prime}(t)\right|+\int_{0}^{T}|e(t)| d t
\end{aligned}
$$

where $\left\|g_{M_{1}}^{+}\right\|:=\max _{d_{2}^{*} \leq x \leq M_{1}} g^{+}(x)$. From Eqs. (2.22) and (4.8), we have

$$
\begin{aligned}
\left\|\phi_{p}\left(x^{\prime}\right)\right\| & \leq \int_{0}^{T}|f(t, x(t))|\left|x^{\prime}(t)\right| d t+\int_{0}^{T}|g(x(t))| d t+\int_{0}^{T}|e(t)| d t \\
& \leq 2\left(T\left\|g_{M_{1}}^{+}\right\|+\int_{0}^{T}|f(t, x(t))|\left|x^{\prime}(t)\right| d t+\int_{0}^{T}|e(t)| d t\right)
\end{aligned}
$$




$$
\begin{aligned}
& \leq 2\left(T\left\|g_{M_{1}}^{+}\right\|+\left\|f_{M_{1}}\right\|_{2}\left(M_{1}^{\prime}\right)^{\frac{1}{2}}+T^{\frac{1}{2}}\|e\|_{2}\right) \\
& :=M_{2}^{\prime}
\end{aligned}
$$

where $f_{M_{1}}:=\max _{0<x(t) \leq M_{1}}|f(t, x(t))|,\left\|f_{M_{1}}\right\|_{2}:=\left(\int_{0}^{T}|f(t, x(t))|^{2} d t\right)^{\frac{1}{2}}$.

Case (II). If $\bar{e}>0$, from (4.7), we have

$$
\int_{0}^{T}|g(x(t))| d t \leq 2 \int_{0}^{T} g^{+}(x(t)) d t+\int_{0}^{T} f(t, x(t)) x^{\prime}(t) d t .
$$

Since $g^{+}(x(t)) \geq 0$, from condition $\left(H_{1}^{*}\right)$, we know that there exists a positive constant $d_{2}^{* *}$ such that $x(t) \geq d_{2}^{* *}$. Therefore, we have

$$
\begin{aligned}
\int_{0}^{T}|g(x(t))| d t & \leq 2 \int_{0}^{T} g^{+}(x(t)) d t+\int_{0}^{T}|f(t, x(t))|\left|x^{\prime}(t)\right| d t \\
& \leq 2 T\left\|g_{M}^{+}\right\|+\int_{0}^{T}|f(t, x(t))|\left|x^{\prime}(t)\right| d t
\end{aligned}
$$

where $\left\|g_{M}^{+}\right\|:=\max _{d_{2}^{* *} \leq x \leq M_{1}} g^{+}(x)$. Similarly, we can get $\left|\phi_{p}\left(x^{\prime}(t)\right)\right| \leq M_{2}^{\prime}$.

The remaining part of the proof is the same as that of Theorem 1.1.

\subsection{Proof of Theorem 1.11}

Proof of Theorem 1.11 We follow the same strategy and notation as in the proof of Theorem 1.5 and 1.6. We can get

$$
x(t) \leq M_{1} .
$$

From Eq. (4.6), we deduce

$$
\begin{aligned}
\int_{0}^{T}|g(x(t))| d t & =\int_{g(x(t)) \geq 0} g(x(t)) d t-\int_{g(x(t)) \leq 0} g(x(t)) d t \\
& =-2 \int_{g(x(t)) \leq 0} g^{-}(x(t)) d t-\int_{0}^{T} f(t, x(t)) x^{\prime}(t) d t+\int_{0}^{T} e(t) d t .
\end{aligned}
$$

Case (I). If $\bar{e}:=\frac{1}{T} \int_{0}^{T} e(t) d t \geq 0$, from Eq. (4.10), we have

$$
\int_{0}^{T}|g(x(t))| d t \leq-2 \int_{0}^{T}\left(g^{-}(x(t))-e(t)\right) d t-\int_{0}^{T} f(t, x(t)) x^{\prime}(t) d t .
$$

Since $g^{-}(x(t))-e(t) \leq 0$, from condition $\left(H_{6}^{*}\right)$, we know $x(t) \geq d_{4}^{*}$. Then we get

$$
\begin{aligned}
\int_{0}^{T}|g(x(t))| d t & \leq-2 \int_{0}^{T} g^{-}(x(t)) d t+\int_{0}^{T}|f(t, x(t))|\left|x^{\prime}(t)\right|+\int_{0}^{T}|e(t)| d t \\
& \leq 2 T\left\|g_{M_{1}}^{-}\right\|+\int_{0}^{T}|f(t, x(t))|\left|x^{\prime}(t)\right|+\int_{0}^{T}|e(t)| d t,
\end{aligned}
$$


where $\left\|g_{M_{1}}^{-}\right\|:=\max _{d_{4}^{*} \leq x \leq M_{1}}\left(-g^{-}(x)\right)$. From Eqs. (2.22) and (4.11), we see that

$$
\begin{aligned}
\left\|\phi_{p}\left(x^{\prime}\right)\right\| & \leq \int_{0}^{T}\left|f(t, x(t)) \| x^{\prime}(t)\right| d t+\int_{0}^{T}|g(x(t))| d t+\int_{0}^{T}|e(t)| d t \\
& \leq 2\left(T\left\|g_{M_{1}}^{-}\right\|+\int_{0}^{T}\left|f(t, x(t)) \| x^{\prime}(t)\right| d t+\int_{0}^{T}|e(t)| d t\right) \\
& \leq 2\left(T\left\|g_{M_{1}}^{-}\right\|+\left\|f_{M_{1}}\right\|_{2}\left(M_{1}^{\prime}\right)^{\frac{1}{2}}+T^{\frac{1}{2}}\|e\|_{2}\right):=M_{2}^{\prime} .
\end{aligned}
$$

Case (II). If $\bar{e}<0$, from Eq. (4.10), we arrive at

$$
\int_{0}^{T}|g(x(t))| d t \leq-2 \int_{0}^{T} g^{-}(x(t)) d t-\int_{0}^{T} f(t, x(t)) x^{\prime}(t) d t .
$$

Since $g^{-}(x(t)) \leq 0$, from condition $\left(H_{6}^{*}\right)$, we know that there exists a positive constant $d_{4}^{* *}$ such that $x(t) \geq d_{4}^{* *}$. Therefore, we conclude that

$$
\begin{aligned}
\int_{0}^{T}|g(x(t))| d t & \leq-2 \int_{0}^{T} g^{-}(x(t)) d t+\int_{0}^{T}|f(t, x(t))|\left|x^{\prime}(t)\right| d t \\
& \leq 2 T\left\|g_{M}^{+}\right\|+\int_{0}^{T}|f(t, x(t))|\left|x^{\prime}(t)\right| d t
\end{aligned}
$$

where $\left\|g_{M}^{-}\right\|:=\max _{d_{4}^{* *} \leq x \leq M_{1}}\left(-g^{-}(x)\right)$. Similarly, we can get $\left|\phi_{p}\left(x^{\prime}(t)\right)\right| \leq M_{2}^{\prime}$.

\section{Examples}

Example 5.1 Consider the following $p$-Laplacian generalized Liénard equation with singularity of attractive type:

$$
\left(\phi_{p}\left(x^{\prime}(t)\right)\right)^{\prime}+\left(\frac{1}{\pi}\left(\cos ^{2} 4 t+3\right) x^{2}+1\right) x^{\prime}(t)-\frac{1}{3 \pi^{2}}(\sin 8 t+2) x^{3}+\frac{1}{x^{\kappa}}=e^{\cos ^{2} 4 t},
$$

where $p=4, \kappa$ is a constant and $\kappa \geq 1$.

Comparing Eq. (5.1) to (1.1), it is easy to see that $f(t, x)=\frac{1}{\pi}\left(\cos ^{2} 4 t+3\right) x^{2}+1, g(t, x)=$ $-\frac{1}{3 \pi^{2}}(\sin 8 t+2) x^{3}+\frac{1}{x^{k}}, e(t)=e^{\cos ^{2} 4 t}, T=\frac{\pi}{4}$. Obviously, there exist constants $d_{3}=0.1$ and $d_{4}=1$ such that $\left(H_{6}\right)$ holds. $1 \leq|f(t, x)| \leq \frac{4}{\pi} x^{2}+1, \alpha=1, m=\frac{4}{\pi}, n=1$, then conditions $\left(H_{2}\right)$ and $\left(H_{5}\right)$ hold. $-g(t, x) \leq \frac{1}{\pi^{2}} x^{3}+1, \beta=\frac{1}{\pi^{2}}, \gamma=1 . \lim _{x \rightarrow 0^{+}} \int_{x}^{1} g_{0}(s) d s=\lim _{x \rightarrow 0^{+}} \int_{x}^{1} \frac{-1}{s^{k}} d s=$ $+\infty$, thus, conditions $\left(H_{7}\right)$ and $\left(H_{8}\right)$ hold. Next, it is verified that

$$
\frac{1}{2^{p-2}}\left(\frac{\beta T}{2}+m\right) T^{\frac{p}{q}}=\frac{1}{2^{2}} \times\left(\frac{1}{2 \pi}+\frac{4}{\pi}\right) \times\left(\frac{\pi}{4}\right)^{3}=\frac{9 \pi^{2}}{2^{9}}<1 .
$$

Therefore, by Theorem 1.5, we know that Eq. (5.1) has at least one positive $\frac{\pi}{4}$-periodic solution.

Example 5.2 Consider the following Liénard equation with singularity of repulsive type:

$$
x^{\prime \prime}(t)+6 x^{10} x^{\prime}(t)+\frac{1}{6 \pi}\left(\sin ^{2} 2 t+5\right) x-\frac{1}{x^{\kappa}}=e^{\cos ^{2} 2 t},
$$

where $p=2, \kappa$ is a constant and $\kappa \geq 1$. 
Comparing Eq. (5.2) to (1.7), it is easy to see that $f(x)=6 x^{10}(t), g(t, x)=\frac{1}{6 \pi}\left(\sin ^{2} 2 t+\right.$ 5) $x-\frac{1}{x^{\kappa}}, e(t)=e^{\cos ^{2} 2 t}, T=\frac{\pi}{2}$. Obviously, there exist constants $d_{1}=0.1$ and $d_{2}=1$ such that $\left(H_{1}\right)$ holds. $g(t, x) \leq \frac{1}{\pi} x+1, a=\frac{1}{\pi}, b=1 . \lim _{x \rightarrow 0^{+}} \int_{x}^{1} g_{0}(s) d s=\lim _{x \rightarrow 0^{+}} \int_{x}^{1} \frac{1}{s^{\kappa}} d s=-\infty$, thus, conditions $\left(H_{3}\right)$ and $\left(H_{4}\right)$ hold. Next, it is verified that

$$
\begin{aligned}
\frac{1}{2^{p-1}} T^{1+\frac{p}{q}} & =\frac{1}{2} \times \frac{1}{\pi} \times\left(\frac{\pi}{2}\right)^{2} \\
& =\frac{\pi}{8}<1 .
\end{aligned}
$$

Therefore, by Theorem 1.6, we know that Eq. (5.2) has at least one positive $\frac{\pi}{2}$-periodic solution.

Example 5.3 Consider the following $p$-Laplacian generalized Liénard equation with singularity of repulsive type:

$$
\left(\phi_{p}\left(x^{\prime}(t)\right)\right)^{\prime}+\left((\cos t+2) x^{6}+3\right) x^{\prime}(t)+\sum_{i=1}^{n} x^{2 i}(t)-\frac{1}{x^{\mu}}=e^{\sin t},
$$

where $p=10, \mu$ is a constant and $\mu \geq 1, n$ is a integer.

Comparing Eqs. (5.3) to (1.8), it is easy to see that $f(t, x)=(\cos t+2) x^{6}+3, g(x)=$ $\sum_{i=1}^{n} x^{2 i}(t)-\frac{1}{x^{\mu}}, e(t)=e^{\cos t}, T=2 \pi$. Obviously, there exist constants $d_{1}^{*}=0.1$ and $d_{2}^{*}=1$ such that $\left(H_{1}^{*}\right)$ holds. $|f(t, x)| \geq 3, \alpha=3$, then condition $\left(H_{2}\right)$ holds. Next, we prove that condition $\left(H_{4}^{*}\right)$ holds. In fact,

$$
\lim _{x \rightarrow 0^{+}} \int_{x}^{1} g(s) d s=\lim _{t \rightarrow 0^{+}} \int_{x}^{1}\left(\sum_{i=1}^{n} s^{2 i}-\frac{1}{s^{\mu}}\right) d s=-\infty .
$$

Therefore, by Theorem 1.9, we know that Eq. (5.3) has at least one positive $2 \pi$-periodic solution.

\section{Conclusions}

In this article we introduce the existence of periodic solution for $p$-Laplacian generalized Liénard equation with singularity of attractive and repulsive type. Due to the friction term $f(t, x) x^{\prime}(t)$ may not satisfy $\int_{0}^{T} f(t, x(t)) x^{\prime}(t) d t=0$. This implies that the work on estimating a priori bounds of periodic solutions for generalized Liénard Eq. (1.1) is more difficult than the corresponding work on Liénard equation in $[6,15,24,25,29]$. Secondly, attractive conditions $\left(H_{7}\right)$ and $\left(H_{8}\right)$ are contradicted with the repulsive conditions $\left(H_{3}\right)$ and $\left(H_{4}\right)$, the methods of $[6,15,24,25,29]$ are no longer applicable to the proof of periodic solution for Eq. (1.1) with singularity of attractive singularity. In this paper, using the ManásevichMawhin theorem on continuity of the topological degree and conditions $\left(H_{1}\right)-\left(H_{5}\right)$, we prove the existence of a periodic solution for equation (1.1) with singularity of repulsive type; by conditions $\left(H_{2}\right),\left(H_{5}\right)\left(H_{6}\right)-\left(H_{8}\right)$, we obtain the existence of a periodic solution for Eq. (1.1) with singularity of attractive type. Moreover, we investigate the existence of a periodic solution for Eqs. (1.7) and (1.8) with singularities of attractive and repulsive type. 
Funding

This work was supported by Education Department of Henan Province project (16B110006) and Henan Polytechnic University Outstanding Youth Fund (J2016-03)

\section{Abbreviation}

Not applicable.

\section{Availability of data and materials}

Not applicable.

\section{Ethics approval and consent to participate}

YX and HML contributed to each part of this study equally and declare that they have no competing interests.

\section{Competing interests}

YX and HML declare that they have no competing interests.

\section{Consent for publication}

$Y X$ and $H M L$ read and approved the final version of the manuscript.

\section{Authors' contributions}

YX and HML contributed equally and significantly in writing this article. Both authors read and approved the final manuscript.

\section{Publisher's Note}

Springer Nature remains neutral with regard to jurisdictional claims in published maps and institutional affiliations.

Received: 19 July 2018 Accepted: 4 December 2018 Published online: 21 December 2018

\section{References}

1. Cheng, Z., Ren, J.: Existence of periodic solution for fourth-order Liénard type p-Laplacian generalized neutral differential equation with variable parameter. J. Appl. Anal. Comput. 5, 704-720 (2015)

2. Cheng, Z., Ren, J.: Periodic solution for second order damped differential equations with attractive-repulsive singularities. Rocky Mt. J. Math. 48, 753-768 (2018)

3. Cheung, W., Ren, J.: Periodic solutions for $p$-Laplacian Liénard equation with a deviating argument. Nonlinear Anal. 59, 107-120 (2004)

4. Cheung, W., Ren, J.: On the existence of periodic solutions for p-Laplacian generalized Liénard equation. Nonlinear Anal. 60, 65-75 (2005)

5. Hakl, R., Zamora, M.: Periodic solution to second-order indefinite singular equations. J. Differ. Equ. 263, 451-469 (2017). https://doi.org/10.1016/j.jde.2017.02.044

6. Jebelean, P., Mawhin, J.: Periodic solutions of forced dissipative p-Liénard equations with singularities. Vietnam J. Math. 32, 97-103 (2004)

7. Kong, F., Lu, S.: Existence of positive periodic solution of fourth-order singular p-Laplacian neutral functional differential equations. Filomat 31, 5855-5868 (2017)

8. Kong, F., Lu, S., Liang, Z.: Periodic solution to second-order indefinite singular equations. Electron. J. Differ. Equ. 2015, $242(2015)$

9. Li, S., Liao, F., Xing, W.: Periodic solutions for Liénard differential equations with singularities. Electron. J. Differ. Equ. 2015, 151 (2015)

10. Liénard, A.: Etude des oscillations entretenues. Rev. Gén. l'Électr. 23, 901-912, 946-954 (1928)

11. Liu, B., Huang, L.: Existence and uniqueness of periodic solutions for a kind of Liénard equation with a deviating argument. Appl. Math. Lett. 21, 56-62 (2008)

12. Liu, W., Feng, Z.: Periodic solutions for p-Laplacian systems of Liénard-type. Commun. Pure Appl. Anal. 10, 1393-1400 (2011)

13. Lu, S., Guo, Y., Chen, L.: Periodic solutions for Liénard equation with an indefinite singularity. Nonlinear Anal., Real World Appl. 45, 542-556 (2019)

14. Lu, S., Jia, X.: Homoclinic solutions for a second-order singular differential equation. J. Fixed Point Theory Appl. 20, Article ID 101 (2018). https://doi.org/10.1007/s11784-018-0575-9

15. Lu, S., Wang, Y., Guo, Y.: Existence of periodic solutions of a Liénard equation with a singularity of repulsive type. Bound. Value Probl. 2017, 95 (2017)

16. Lu, S., Zhong, T., Chen, L.: Periodic solutions for p-Laplacian Rayleigh equations with singularities. Bound. Value Probl. 2016, 96 (2016)

17. Ma, T., Wang, Z.: Periodic solutions of Liénard equations with resonant isochronous potentials. Discrete Contin. Dyn. Syst. 33, 1563-1581 (2013)

18. Manásevich, R., Mawhin, J.: Periodic solutions for nonlinear systems with p-Laplacian-like operator. J. Differ. Equ. 145 367-393 (1998). https://doi.org/10.1006/jdeq.1998.3425

19. Mawhin, J.: Periodic solutions for quasilinear complex-valued differential systems involving singular $\phi$-Laplacians. Rend. Ist. Mat. Univ. Trieste 44, 75-87 (2012)

20. Torres, P.: Nondegeneracy of the periodically forced Liénard differential equation with p-Laplacian. Commun. Contemp. Math. 13, 283-292 (2011)

21. Villari, G.: An improvement of Massera's theorem for the existence and uniqueness of a periodic solution for the Liénard equation. Rend. Ist. Mat. Univ. Trieste 44, 187-195 (2012) 
22. Wang, Y., Cheng, S., Ge, W.: Periodic solutions of generalized Liénard equations with a p-Laplacian-like operator. Bull. Braz. Math. Soc. 39, 21-43 (2008)

23. Wang, Y., Dai, X., Xia, X.: On the existence of a unique periodic solution to a Liénard type p-Laplacian non-autonomous equation. Nonlinear Anal. TMA 71, 275-280 (2009)

24. Wang, Z: Periodic solutions of Liénard equation with a singularity and a deviating argument. Nonlinear Anal., Real World Appl. 16, 227-234 (2014)

25. Xin, Y., Cheng, Z:: Study on a kind of p-Laplacian Liénard equation with attractive and repulsive singularities. J. Inequal. Appl. 2017, 180 (2017)

26. Xin, Y., Han, X., Cheng, Z.: Existence and uniqueness of positive periodic solution for p-Laplacian Liénard equation. Bound. Value Probl. 2014, 244 (2014)

27. Yang, L., Zeng, X.: The period function of Liénard systems. Proc. R. Soc. Edinb., Sect. A 143, 205-221 (2013)

28. Zamora, M.: On a periodically forced Liénard differential equation with singular p-Laplacian. Bull. Math. Soc. Sci. Math. Roum. 57, 327-336 (2014)

29. Zhang, M.: Periodic solutions of Liénard equations with singular forces of repulsive type. J. Math. Anal. Appl. 203, 254-269 (1996)

30. Zhang, Z., Yuan, R.: Existence of positive periodic solutions for the Liénard differential equations with weakly repulsive singularity. Acta Appl. Math. 111, 171-178 (2010)

Submit your manuscript to a SpringerOpen ${ }^{\circ}$ journal and benefit from:

- Convenient online submission

- Rigorous peer review

- Open access: articles freely available online

- High visibility within the field

Retaining the copyright to your article

Submit your next manuscript at $\boldsymbol{~ s p r i n g e r o p e n . c o m ~}$ 